\title{
A Phase II Study of Antineoplastons A10 and AS2-1 in Adult Patients with Recurrent Glioblastoma Multiforme: Final Report (Protocol BT-21)
}

\author{
Stanislaw R. Burzynski*, Tomasz J. Janicki, Gregory S. Burzynski \\ Burzynski Clinic, Houston, Texas, USA \\ Email: ${ }^{*}$ srb@burzynskiclinic.com
}

Received 11 July 2014; revised 5 August 2014; accepted 1 September 2014

Copyright (C) 2014 by authors and Scientific Research Publishing Inc.

This work is licensed under the Creative Commons Attribution International License (CC BY). http://creativecommons.org/licenses/by/4.0/

(c) (i) Open Access

\section{Abstract}

Treatment of recurrent glioblastoma multiforme (RGBM) creates one of the most difficult challenges to neuro-oncology. The purpose of this study is to evaluate the outcome of adults with high-grade glioma with special attention to RGBM patients treated with Antineoplastons (ANP) A10 and AS2-1 injections. The study was conducted according to Protocol BT-21, which accrued patients who failed standard radiation therapy (RT) and chemotherapy. There were 40 candidates registered in the study. Among the intent-to-treat (ITT) population, there were 30 cases of RGBM that progressed during and after prior treatment, 4 patients with anaplastic astrocytoma (AA), 1 with anaplastic mixed glioma (AMG), and 5 with persistent GBM. The aim of this paper is to evaluate the responses, survival and toxicity of all 40 patients, the efficacy in 30 patients with RGBM, and in 24 patients with RGBM who received at least 28 days of ANP (ERGBM). All RGBM patients were treated before with RT and chemotherapy, except one patient who only had surgery (patient refused radiation). In this group, $63 \%$ had one recurrence, $30 \%$ had two recurrences, and $7 \%$ had three recurrences. The median duration of ANP and ITT was 12 weeks and the median dosage of ANP A10 was $6.52 \mathrm{~g} / \mathrm{kg} / \mathrm{d}$ and ANP AS2-1 was $0.23 \mathrm{~g} / \mathrm{kg} / \mathrm{d}$. Responses were assessed by gadolinium-enhanced magnetic resonance imaging (MRI) repeated every eight weeks. In the ITT population, objective responses (ORs) were determined in $10 \%$ of cases (complete response-CR, and partial response-PR in 5\% each). Progression-free survival (PFS) in ITT at six months was $17.5 \%$. Overall survival (OS) was $28.3 \%$ at one year, $2.6 \%$ at two years, five and ten years. In the RGBM population, objective responses (ORs) were determined in $13.3 \%$ of cases (CR and PR in $6.7 \%$ each). PFS in RGBM at six months was $16.7 \%$. OS was $34.7 \%$ at one year, $3.47 \%$ at two years, five and ten years. In the ERGBM population, ORs were determined in $16.7 \%$ of cases (CR and PR in $8.3 \%$ each). PFS in ERGBM at six months was $20.8 \%$, OS was $39.3 \%$ at one year, $4.4 \%$ at two years, five and ten years. The treatment was well-tolerated with reversible Grades 3 and 4 toxicity in

\footnotetext{
${ }^{*}$ Corresponding author.
} 
$17.5 \%$ of patients ( 7 patients who experienced multiple toxicities) and no chronic toxicity. In conclusion, the study reached efficacy endpoint. ANP is well-tolerated and compares favorably to the current treatment for RGBM.

\section{Keywords}

\section{Antineoplastons A10 and AS2-1, Recurrent Glioblastoma Multiforme, Phase II Clinical Trial, Survival in Glioblastoma}

\section{Introduction}

Glioblastoma multiforme (GBM) is the most common highly-malignant brain tumor with an average annual incidence in the U.S. of over 50,000 cases [1]. It is estimated that over $90 \%$ of cases of this patient population will develop recurrence of the disease (RGBM). It is currently thought that GBM develops through the progressive accumulation of epigenetic and genetic changes that help the cells to escape destruction by molecular mechanisms of the body [2]. Hereditary factors seem to contribute to less than 1\% of GBMs [3]. On the other hand, there is vast literature indicating that environmental exposure is associated with increased incidence of GBM. Ionizing radiation is the only environmental factor that is consistently associated with the development of GBM [2] [4]. Chemical exposure as a possible cause of glioma is an actively debated issue for over 30 years [5]. A number of chemicals have been identified that induce brain tumors in animals, but the evidence from the epidemiological studies are not conclusive [2] [6]-[8]. The interesting results were found in epidemiologic studies of association between allergies and cancer [9]. Allergic condition appeared to increase the risk of various types of cancer, but there is documented inverse relationship between allergies, asthma, and immunoglobulin E levels and risk and prognosis of glioma [10]. GBM has inherent feature to progress due to accumulation of epigenetic and genetic abnormalities over time, which is similar to other aggressive types of cancer [11]. The main difference between these cancers and GBM is an extremely complex network of abnormal genes in GBM [12]. Unstable GBM genome undergoes continuous changes and converts to RGBM after standard-of-care treatment [13] [14].

Standard of care treatment for newly-diagnosed GBM consists of surgical resection followed by radiotherapy (RT) with concurrent temozolomide (TMZ) and six maintenance cycles of adjuvant TMZ [15]. The improvement in overall survival in such treatment is mostly restricted to a subgroup of patients with silenced DNA repair gene 0-6-methylguanine-DNA methyltransferase (MGMT) [16]. MGMT promoter methylation seems to be associated with improvement of progression-free survival (PFS) (8.7 vs. 5.7 months) and overall survival (OS) (21.2 vs. 14 months) [17]. Unfortunately, over $90 \%$ of patients suffer recurrence [18]. A small number of patients with recurrent glioblastoma multiforme (RGBM) may undergo a second surgery or RT. In patients who relapsed after TMZ, 20\% PFS at 6 months can be accomplished with nitrosoureas, TMZ in different dosing regimens or bevacizumab [19] [20]. It is a general consensus that more research is needed to introduce better treatment regimens for patients with RGBM [19].

ANPs are synthetic derivatives of glutamine, isoglutamine, and phenylacetic acid [21]. The initial studies revealed objective responses and long-term OS in patients diagnosed with GBM [22]. With positive preliminary data, we have designed and conducted a single-arm Phase II study of ANP A10 and AS2-1 in patients with recurrent high-grade glioma with special emphasis on RGBM.

\section{Patients and Methods}

\subsection{Patient Population}

The inclusion criteria of the protocol required the age of the patients to be over 18 years, a resected or biopsied tumor prior to RT, and radiologic evidence of recurrent tumor by gadolinium-enhanced magnetic resonance imaging (MRI) performed within 14 days before initiating the study. It was requested that there should be a complete recovery and at least four weeks from prior surgical procedure. The Karnofsky Performance Status (KPS) should be between 60 and 100, hemoglobin concentration of at least $10 \mathrm{~g} / \mathrm{dl}$, a white blood count of at least 
$1500 / \mathrm{mm}^{3}$, and a platelet count of at least $50,000 / \mathrm{mm}^{3}$. The subjects' total bilirubin and serum creatinine concentrations should be no higher than $2.5 \mathrm{mg} / \mathrm{dL}$, and aspartate aminotransferase (AST) and alanine aminotransferase (ALT) no higher than five times the upper limits of normal. The exclusion criteria did not permit admission of pregnant or breast-feeding females, patients with high medical or psychiatric risks due to non-malignant disease, presence of active infection, chronic heart failure, serious lung disease, uncontrolled hypertension and inadequate hepatic function, brainstem location of the tumor and patients incompetent to give informed consent for treatment. The use of corticosteroids was permitted to reduce symptoms and signs attributed to cerebral edema, but it was recommended that the smallest doses compatible with the preservation of optimal neurologic function, be used. Confirmation of the pathologic diagnosis by an outside pathologist was also required. Patients were removed from the study if 1) progressive disease (PD) developed, 2) if toxicity levels became unacceptable, 3 ) if the subject developed a concurrent illness that interfered with therapy, 4) if the subject or guardian asked to be removed from the study or became non-compliant with the study criteria, and 5) if the subject completed at least 8 months of treatment after determination of complete response (CR), partial response (PR) or stable disease (SD). All study subjects and/or guardians read, understood, and signed written informed consent prior to enrollment. This study was conducted in accordance with the U.S. Code of Federal Regulations, Title 21, Parts 11, 50, 56, and 312; the Declaration of Helsinki (1964) including all amendments and revisions; the Good Clinical Practices: Consolidated Guideline (E6); International Conference on Harmonization; and the FDA's Guidance for Industry. The study was sponsored by the Burzynski Research Institute, Inc., (BRI) and conducted by the Burzynski Clinic (BC) in Houston, Texas. The patients did not pay for the investigational agents.

\subsection{Study Design}

The study was designed as a single-arm, two-stage, interventional Phase II trial of ANP A10 and AS2-1 as the single therapy modality in a high-risk, poor-prognosis study population [23]. The study was listed by the National Cancer Institute (NCI). It was supervised by the independent Institutional Review Board (BRI-IRB, BCBT-21). The study was performed according to Protocol BT-21 which was submitted to the FDA under the IND 43,742. The study commenced on February 26, 1996, and closed to accrual on February 25, 2011. The protocol was amended by BRI several times; however, none of the amendments altered the aim or design of the original study objectives/outcomes.

\section{Statistical Consideration}

The sample size and statistical methods were based upon the method described by Chang et al. and were described before [24]. A response rate to ANP of $\geq 10 \%$ was considered "of interest", and the primary endpoint was to determine the overall response rate (OR = CR + PR) to ANP therapy. An interim analysis would be conducted after 20 subjects had enrolled in the study. If 1 or more patients achieved a confirmed radiographic response, an additional 20 subjects would be recruited. OS was measured from the first day of ANP administration until death from any cause, and progression-free survival (PFS) was measured from the first day of the treatment until the date of first observation of PD, beginning of other treatment or death. OS and PFS were estimated by Kaplan-Meier analysis using the MedCalc Statistical Software version 13.3 (MedCalc Software bvba, Ostend, Belgium; 2014. The primary endpoint was the response to treatment which was determined in these different populations: 1) intent-to-treat (ITT) population, 2) RGBM, and 3) RGBM patients who received at least 28 days of ANP (ERGBM). Time to best response, dosage, and duration of treatment were analyzed. The average maximum effective daily dose and the range were calculated.

\section{Treatment Plan}

ANP A10 and AS2-1 were the only anti-tumor treatments administered in this study. The formulations were delivered via a dual-channel infusion pump and single-lumen subclavian catheter (Broviac or Groshong) every 4 hours. A loading dose of $72 \mathrm{~g}$ of A10 in a fluid formulation of concentration $0.3 \mathrm{gram} / \mathrm{mL}$ and $16 \mathrm{~g}$ of AS2-1 in a fluid formulation of $0.08 \mathrm{~mL}$ was distributed evenly into 6 single doses after an initial testing dose of $10 \mathrm{~mL}$ of each. The next day the dose was increased by $72 \mathrm{~g}$ and $16 \mathrm{~g}$ respectively and the escalation was repeated daily until maximum tolerated dose was established. When the study subject reached the highest tolerated dose, but not higher than $20 \mathrm{~g} / \mathrm{kg} /$ day of A10 and $0.4 \mathrm{~g} / \mathrm{kg} /$ day of AS2-1 the "escalation phase" of the treatment stopped and the subject continued the treatment which required daily administration of 6 doses of A10 and AS2-1 (every 
4 hours). The study subject continued the established dose until a response to the treatment was determined, and it was advised to continue treatment for at least eight months after CR was documented. Dosing was stopped at patient request or if their clinical condition worsened. After discontinuation of IV ANP, the patients were eligible for a maintenance treatment with $0.5 \mathrm{~g}$ capsules of A10 and AS2-1. According to the maintenance plan, patients were taking up to $2.5 \mathrm{~g}$ six times a day of both A10 and AS2-1. The escalation of the dosage of ANP was required based on the results of prior studies [21] to find out if the patients were able to tolerate large volume infusions of intravenous fluids associated with higher doses of ANP. As a safety precaution it was recommended that the escalation of the dosages will continue through Phase II and Phase III trials programs. Medications that were considered necessary for the subjects' welfare and that did not interfere with the evaluation of treatment were given at the discretion of the investigator. The use of corticosteroids was carefully monitored. Subjects received full supportive care, including transfusions of blood products and antibiotics when appropriate. No other anticancer mediation was permitted.

The initial two weeks of therapy was administered by BC staff on an outpatient basis. Subjects and/or their legal guardians were trained by radiological clinic staff to self-administer ANP therapy. Based on prior experience, starting on week 3 ANP therapy was administered by the enrollee or their guardian at home. Treatment and monitoring of the subject's condition, once released to self-administered therapy, continued under the supervision of the subject's local physician.

\section{Evaluation and Follow-Up}

Prior to the start of treatment, a gadolinium-enhanced magnetic resonance imaging (MRI) measured all contrast-enhancing lesions. The products of the two largest perpendicular diameters (LPD) of all lesions were calculated and totaled, providing a baseline evaluation for each study subject. As a common practice at that time in other clinical trials, the tumor measurements were based on contrast enhanced lesions, but the overall tumor size was also measured including T2 and FLAIR images [19] [23]. The baseline provided the reference for determining response outcomes to the treatment. Blood and urine tests (complete blood count with differential, platelet count, reticulocyte count, and serum chemistry) anticonvulsant serum levels, prothrombin time, and partial thromboplastin time were carried out on all subjects prior to the start of treatment to establish normal baselines. The additional pretreatment measurements included KPS, vital signs, clinical disease status, demographics, medical history and current medications, physical examination with neurologic emphasis, chest X-rays and EKG. Toxicity was evaluated in ITT population. Data on adverse experiences (AEs) were collected during the initial 3 weeks of ANP therapy by clinic staff at subject request. In accordance with other Phase II studies conducted at the initiation of this trial, the possible responses to the treatment were CR, PR, SD and PD. CR required the disappearance of all enhancing lesions, sustained for at least 4 weeks, and only physiologic replacement doses of steroids were acceptable. PR required 50\% or higher decrease of the LPD of enhancing lesions and stable or reduced corticosteroid doses. PD was determined when there was over $50 \%$ increase of enhancing lesions or new lesions, and SD was the status between PR and PD. The results of all MRI and PET scans were verified by radiologists not affiliated with BRI or BC and their determination of response was accepted. Study subjects were categorized by their overall best response during the course of the treatment. The duration of each response was measured from the date that the criteria for the outcome were first met until the date that PD was first documented. The original protocol required more than 50\% increase of tumor size for PD, but in this publication $25 \%$ increase was acceptable and all cases reported in this paper were re-evaluated by radiological RANO criteria to allow comparison with other studies. In the case of SD, the duration was measured from the time therapy commenced. During the study the generally accepted criteria for evaluation of responses in high-grade glioma changed toward reliance on OS and PFS rather than tumor responses. As a result, the protocol was amended to include also survival analysis. Correlative studies including $1 p 19 q$ deletions and MGMT silencing were not performed because they were not yet introduced when the patients were accrued except for the three patients who were accrued before completion of the study.

\section{Results}

\subsection{Patient Demographic}

Forty candidates were registered in the study (ITT population). There were 35 cases of GBM, 4 cases of anaplastic astrocytoma (AA) and 1 case of anaplastic mixed glioma (AMG). The characteristics of the patients are 
listed in Table 1.

Among 35 patients diagnosed with GBM, there were 30 cases of RGBM and 5 cases of persistent GBM. In the group of 30 RGBM patients, there were 24 subjects who received at least 28 days of ANP (ERGBM). The characteristics of GBM patients are presented in Table 2.

Among three patients who had genomic analyses, none of them had IDH1 mutation. One of these patients could be assigned to the subgroup of classical GBM [12]. He had amplified EGFR and silenced MGMT and developed PD during the treatment with ANP. Two remaining patients had reduced, but active MGMT and one of them could be assigned to a proneural subgroup. Both of them obtained stabilization of disease.

\subsection{Treatment}

In the ITT group the median daily dose of ANP A10 ranged from 1.27 to $11.88 \mathrm{~g} / \mathrm{kg} / \mathrm{d}$ with a median of 6.52 $\mathrm{g} / \mathrm{kg} / \mathrm{d}$. For AS2-1, the median daily dose was $0.23 \mathrm{~g} / \mathrm{kg} / \mathrm{d}$, with a range of 0.11 to $0.38 \mathrm{~g} / \mathrm{kg} / \mathrm{d}$. The median daily

\section{Table 1. Study population demographics—study BT-21.}

\begin{tabular}{|c|c|c|}
\hline \multicolumn{3}{|c|}{$\begin{array}{c}\text { ENROLLED SUBJECTS } \\
\mathbf{N}=\mathbf{4 0}\end{array}$} \\
\hline Characteristic & $\mathbf{N}$ & $\%$ \\
\hline \multicolumn{3}{|l|}{ Age (years) } \\
\hline Median & 48 & \\
\hline Range & $19-68$ & \\
\hline \multicolumn{3}{|l|}{ Sex } \\
\hline Male & 26 & 65 \\
\hline Female & 14 & 35 \\
\hline \multicolumn{3}{|c|}{ Karnofsky performance score } \\
\hline Median & 70 & \\
\hline Range & $60-90$ & \\
\hline \multicolumn{3}{|c|}{ Diagnosis } \\
\hline GBM & 35 & 87.5 \\
\hline AA & 4 & 10 \\
\hline AMG & 1 & 2.5 \\
\hline \multicolumn{3}{|c|}{ Clinical characteristics } \\
\hline Recurrent tumors & 34 & 85 \\
\hline Persistent tumors & 6 & 15 \\
\hline \multicolumn{3}{|c|}{ Prior treatment characteristics } \\
\hline Surgery only & 2 & 5 \\
\hline RT only & 5 & 12.5 \\
\hline Surgery, RT, and CH & 16 & 40 \\
\hline Surgery and RT & 9 & 22.5 \\
\hline Surgery and $\mathrm{CH}$ & 3 & 7.5 \\
\hline $\mathrm{RT}$ and $\mathrm{CH}$ & 5 & 12.5 \\
\hline
\end{tabular}

AA—anaplastic astrocytoma; AMG—anaplastic mixed glioma; GBM—glioblastoma multiforme; RT—radiation therapy; $\mathrm{CH}$-chemotherapy. 
Table 2. Characteristics of glioblastoma multiforme patients—study BT-21.

\begin{tabular}{|c|c|c|}
\hline \multicolumn{3}{|c|}{ Clinical characteristics of GBM ( $=35)$} \\
\hline & $\mathbf{N}$ & $\%$ \\
\hline Recurrent GBM & 30 & 86 \\
\hline Persistent GBM & 5 & 14 \\
\hline \multicolumn{3}{|c|}{ Prior treatment in RGBM $(\mathrm{N}=30)$} \\
\hline \multicolumn{3}{|c|}{ Number of recurrences } \\
\hline 1 & 19 & 63 \\
\hline 2 & 9 & 30 \\
\hline 3 & 2 & 7 \\
\hline \multicolumn{3}{|c|}{ Prior treatment in ERGBM $(\mathrm{N}=24)$} \\
\hline \multicolumn{3}{|c|}{ Number of recurrences } \\
\hline 1 & 15 & 63 \\
\hline 2 & 8 & 33 \\
\hline 3 & 1 & 4 \\
\hline
\end{tabular}

ERGBM—eligible recurrent glioblastoma multiforme; GBM—glioblastoma multiforme; RGBM—recurrent glioblastoma multiforme.

dose of A10 in subjects until first OR was $4.97 \mathrm{~g} / \mathrm{kg} / \mathrm{d}$; (range 4.59 to $6.99 \mathrm{~g} / \mathrm{kg} / \mathrm{d}$ ) and the median daily dose of AS2-1 was $0.26 \mathrm{~g} / \mathrm{kg} / \mathrm{d}$; (range 0.22 to $0.28 \mathrm{~g} / \mathrm{kg} / \mathrm{d}$ ). The median time to first OR was 46.5 weeks (range 37.1 to 58.6 weeks). In the group of subjects with an SD response, the median daily dose of A10 was $7.14 \mathrm{~g} / \mathrm{kg} / \mathrm{d}$ (range 3.67 to $9.59 \mathrm{~g} / \mathrm{kg} / \mathrm{d}$ ) and the median daily dose of AS2-1 was $0.26 \mathrm{~g} / \mathrm{kg} / \mathrm{d}$ (range 0.22 to $0.38 \mathrm{~g} / \mathrm{kg} / \mathrm{d}$ ). The duration of IV ANP therapy ranged from 0.4 to 58.6 weeks with a median of 11.9 weeks. One PR patient from RGBM group was eligible for a maintenance oral treatment of A10 and AS2-1 capsules. One additional SD patient from the persistent GBM group was given maintenance oral treatment. In the RGBM group, the median daily dose of ANP A10 ranged from 1.27 to $11.88 \mathrm{~g} / \mathrm{kg} / \mathrm{d}$ with a median of $6.95 \mathrm{~g} / \mathrm{kg} / \mathrm{d}$. For AS2-1, the median daily dose was $0.24 \mathrm{~g} / \mathrm{kg} / \mathrm{d}$, with a range of 0.15 to $0.38 \mathrm{~g} / \mathrm{kg} / \mathrm{d}$. The median daily dose of A10 and AS2-1 for OR is the same for ITT and RGBM. The median duration to the first OR was 10.9 weeks (range from 0.43 to 58.3 weeks). One SD RGBM patient received a median dose of A10 9.74 g/kg/d and AS2-1 0.26 g/kg/d. There was no statistical difference from the ERGBM group and the RGBM group when comparing the median daily doses and time to first OR, since ERGBM is a subgroup of RGBM.

\section{Responses and Survival}

The responses were evaluated separately for all patients (ITT), for the group of RGBM and for ERGBM. Four patients obtained OR (10\%, 13.3\% and 16.7\% for ITT, RGBM and ERGBM respectively). The responses of two of them were classified as CR, and the other two as PR (Figure 1).

Four patients in the ITT population obtained SD (10\%), and 19 patients (47.5\%) developed PD. Thirteen patients (32.5\%) were nonevaluable since they did not have follow-up evaluation or did not complete twelve weeks of therapy without progression. Clinical benefit (CR + PR + SD) was seen in 8 patients (20\%). One CR patient received treatment with ANP after he relapsed after surgical resection, RT and chemotherapy with Glia$\mathrm{del}^{\circledR}$ wafers. This patient developed recurrence after 2.5 years of CR after ANP treatment and underwent surgical resection. His tumor transformed to primitive neuroectodermal tumor (PNET) for which he received further treatment (Figure 1). He is currently well and alive over 12 years after treatment start. Another CR case came for treatment with ANP after his tumor recurred after two different types of RT and TMZ. Among two PR cases, one developed tumor recurrence after surgical resection and two different types of RT, and another patient was treated with ANP after surgical resection, RT and chemotherapy. In RGBM group, SD was 3.3\%, PD was 50\%, and NE was 33.3\%. In ERGBM group, SD was 4.2\%, PD was 58.3\%, and NE was 20.8\%. 
In the ITT group, OS was $48.9 \%$ at 6 months, $28.3 \%$ at 1 year and $2.6 \%$ at two, five and ten years. The PFS at six months was $17.5 \%$. In the RGBM group, OS was $55.5 \%$ at 6 months, $34.7 \%$ at 1 year and $3.5 \%$ at two, five and ten years. The PFS at six months was $16.7 \%$. In the ERGBM group, OS was $65.5 \%$ at 6 months, $39.3 \%$ at 1 year and $4.4 \%$ at two, five and ten years. The PFS at six months was 20.8\% (Figure 2 and Figure 3).

Table 3 compares the results of the current study to other select studies.

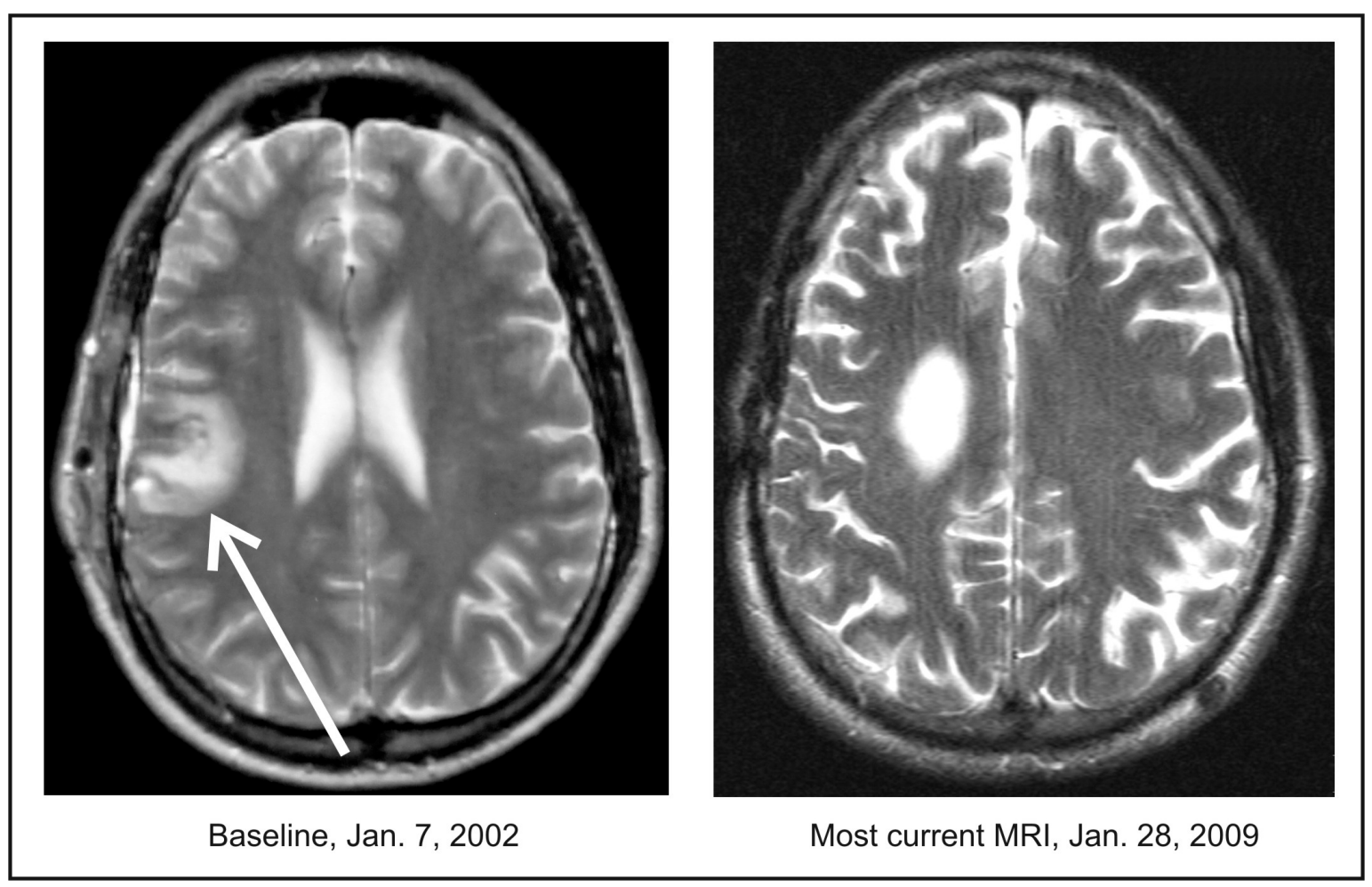

Figure 1. Patient 28. A 42-year-old male diagnosed with a GBM developed recurrence after gross total resection, RT and chemotherapy with Gliadel ${ }^{\circledR}$ wafers. The patient was admitted to the Phase II study on January 7, 2002 and discontinued treatment on March 1, 2003. The CR was documented on February 13, 2002. OS is over 12 years.

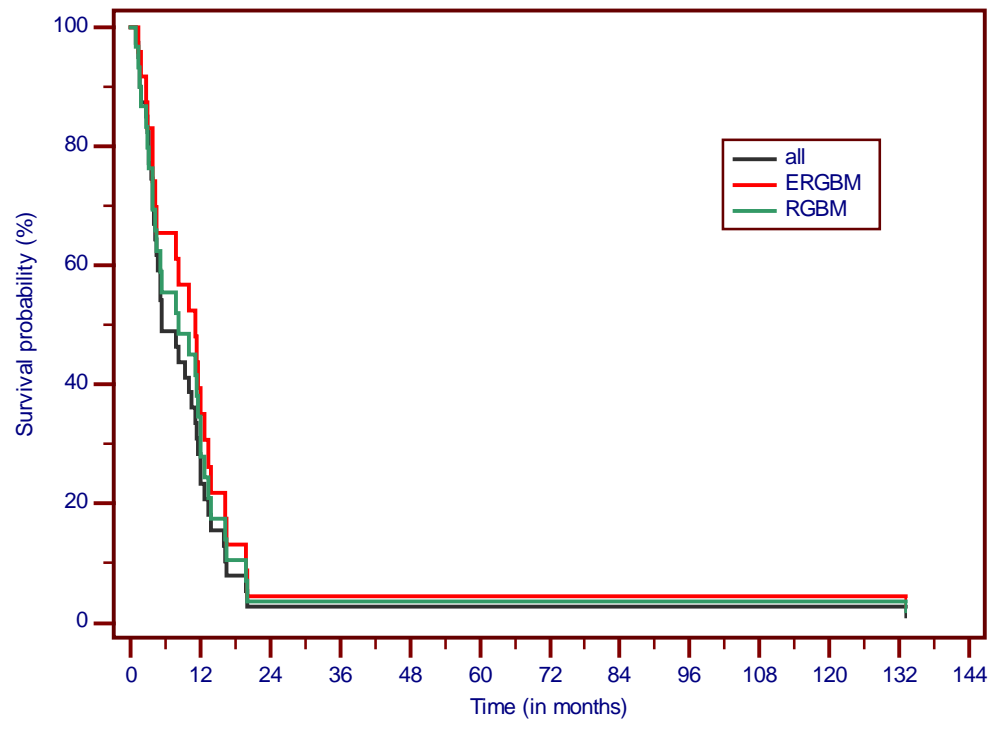

Figure 2. The Kaplan-Meier survival curve. Overall survival from the treatment start. 


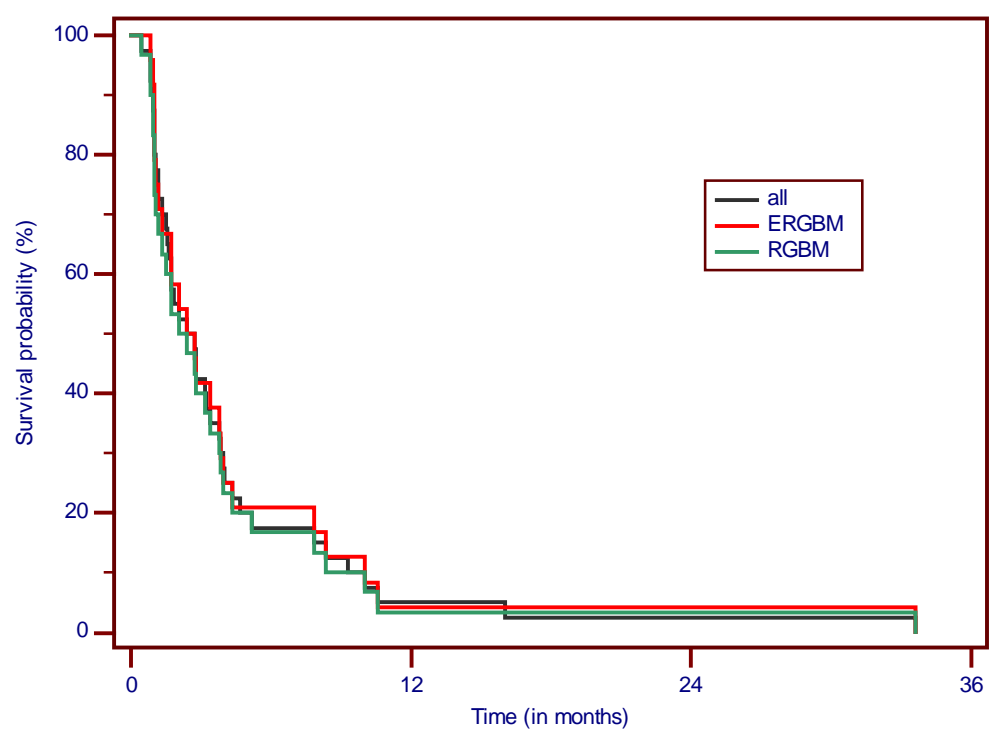

Figure 3. Progression-free survival.

\section{Safety and Adverse Events}

\section{Toxicity}

The following serious adverse drug events (ADEs) were observed during the course of treatment in the ITT group: hypernatremia $12.5 \%$, hypokalemia $7.5 \%$, fatigue $5 \%$, and somnolence $2.5 \%$. All serious ADEs were observed in a group of seven patients. Generally, the treatment was well-tolerated with reversible toxicities. There were no chronic toxicities. All adverse events (AEs) have been coded according to Common Terminology Criteria for Adverse Events v3 (CTCAEv3). Table 4 compares incidence of ADEs in this study to other studies of chemotherapy for RGBM. It is planned to decrease the maximum dosage of ANP A10 in future studies to 12 $\mathrm{g} / \mathrm{kg} / \mathrm{d}$ to reduce the incidence of the most important ADEs: hypernatremia, hypokalemia, fatigue and somnolence.

\section{Discussion}

Clinical trials in RGBM have been extensively reviewed [19]. Unfortunately, at recurrence only a small number of patients were eligible for second surgery or RT. In patients who received prior TMZ, a PFS at 6 months is between $20 \%$ - 30\% after retreatment with TMZ in different dosing regimens, nitrosoureas, or bevacizumab. Regimens with newer targeted therapy did not produce evidence of greater activity, but were usually associated with more toxicity. The treatment with ANP for RGBM and ERGBM was compared with the results of four other Phase II studies in which GBM recurred after RT and chemotherapy with TMZ (in one study TMZ and bevacizumab) (Table 3).

Vredenburgh et al. conducted Phase II of bevacizumab and irinotecan in recurrent malignant glioma including 23 patients with GBM. He reported 4\% of CR, 56\% PR and 38\% SD [25]. Despite remarkable objective responses, 6-month PFS was 30\%, median PFS 4.6 months, and median OS 9.3 months. The toxicity was substantial and $9 \%$ of patients died as the result of ADE (Table 4).

Reardon et al. reported the results of Phase II study of carboplatin, irinotecan and bevacizumab for recurrent GBM after progression on TMZ and bevacizumab [26]. Nearly all patients were enrolled while progressing on bevacizumab therapy. There was no CR or PR, but $80 \%$ of patients had SD. PFS at 6 months was $16 \%$, median PFS 2.3 months, and median OS 5.8 months. A substantial percent of patients experienced thrombocytopenia and neutropenia. Desjardins et al. conducted treatment with daily TMZ for recurrent GBM in 32 patients [27]. The authors reported 28\% PR and 30\% SD; however, PFS at 6 months was 18.8\%, median PFS was 3.6 months, and median OS only 8.6 months. One-year survival was $31 \%$ and two-year survival $8 \%$. The toxicity was tolerable, but there was one treatment-related death. Omura et al. reported the results of Phase II study of continuous low-dose TMZ in 28 patients with RGBM who relapsed during prior treatment [20]. There was no CR, but 11\% 
Table 3. Selected Phase II clinical studies in recurrent glioblastoma multiforme in comparison to results of current study.

\begin{tabular}{|c|c|c|c|c|c|c|c|c|c|c|c|c|}
\hline \multirow{3}{*}{ Reference/Treatment } & \multirow{3}{*}{$\begin{array}{c}\text { Patients } \\
\text { Total } \\
\text { No. }\end{array}$} & \multirow{2}{*}{\multicolumn{2}{|c|}{ Prior treatment }} & \multirow{2}{*}{\multicolumn{2}{|c|}{$\begin{array}{c}\text { Efficacy } \\
\text { response } \\
(\%)\end{array}$}} & \multicolumn{7}{|c|}{ Survival analysis } \\
\hline & & & & & & \multicolumn{2}{|c|}{ PFS } & \multicolumn{5}{|c|}{ Overall survival } \\
\hline & & $\begin{array}{c}\text { Radiation } \\
\text { Therapy and } \\
\text { Chemotherapy \% }\end{array}$ & Chemotherapy \% & CR & PR & $\begin{array}{c}\text { Median } \\
(\mathrm{mos})\end{array}$ & $\begin{array}{c}6 \mathrm{mos} \\
\%\end{array}$ & $\begin{array}{l}\text { Median } \\
\text { (mos) }\end{array}$ & $\begin{array}{c}6 \mathrm{mos} \\
\%\end{array}$ & $\begin{array}{c}1 \mathrm{y} \\
\%\end{array}$ & $\begin{array}{l}2 \mathrm{y} \\
\%\end{array}$ & $\begin{array}{c}10 y \\
\%\end{array}$ \\
\hline $\begin{array}{c}\text { Vredenburgh et al., } 2007 \\
\text { Bevacizumab and irinotecan [25] }\end{array}$ & 23 & All & TMZ-All & 4 & 56 & 4.6 & 30 & 9.3 & & & & \\
\hline $\begin{array}{c}\text { Reardon et al., } 2011 \\
\text { Carboplatin, irinotecan and } \\
\text { bevacizumab [26] }\end{array}$ & 25 & All & $\begin{array}{c}\text { TMZ-All } \\
\text { Bevacizumab }\end{array}$ & 0 & 0 & 2.3 & 16 & 5.8 & & & & \\
\hline $\begin{array}{c}\text { Desjardins et al., } 2012 \\
\text { Bevacizumab and daily TMZ [27] }\end{array}$ & 32 & All & TMZ-All & 0 & 28 & 3.6 & 18.8 & 8.6 & & 31 & 8 & \\
\hline $\begin{array}{l}\text { Omuro et al., } 2013 \\
\text { Low-dose TMZ [20] }\end{array}$ & $28^{*}$ & All & TMZ-All & 0 & 11 & 2 & 19 & 7 & & 35 & & \\
\hline Burzynski et al., 2014 & & & & & & & & & & & & \\
\hline $\begin{array}{c}\text { Antineoplastons A10 and AS2-1 } \\
\text { RGBM }\end{array}$ & 30 & $57^{* *}$ & 10 & 6.7 & 6.7 & 2.1 & 16.7 & 8.3 & 55.5 & 34.7 & 3.5 & 3.5 \\
\hline $\begin{array}{c}\text { Burzynski et al., } 2014 \\
\text { Antineoplastons A10 and AS2-1 } \\
\text { ERGBM }\end{array}$ & 24 & $62.5^{* * *}$ & 25 & 8.3 & 8.3 & 2.4 & 20.8 & 11.5 & 65.5 & 39.3 & 4.4 & 4.4 \\
\hline
\end{tabular}

CR — complete response; ERGBM — eligible recurrent glioblastoma multiforme; OS—overall survival; PR—partial response; PFS—progression-free survival; RGBM-recurrent glioblastoma multiforme; SD—stable disease; TMZ_temozolomide. ${ }^{*} 28$ evaluable patients, 37 included in survival analysis; ${ }^{* *}$ The additional $30 \%$ of patients were treated with RT only and $3 \%$ of patients underwent surgery only; ${ }^{* * *}$ The additional $12.5 \%$ of patients were treated with RT only.

Table 4. Incidence of adverse drug experiences (ADE), grades 3 and 4 and treatment related deaths reported in antineoplastons study (ITT) compared to the studies of chemotherapy for recurrent glioblastoma multiforme.

\begin{tabular}{|c|c|c|c|c|c|}
\hline $\begin{array}{c}\text { ADE } \\
\text { (Incidence \%) }\end{array}$ & Antineoplastons & $\begin{array}{l}\text { Vredenburgh } \\
\text { et al. }[25]\end{array}$ & $\begin{array}{l}\text { Reardon } \\
\text { et al. [26] }\end{array}$ & $\begin{array}{l}\text { Desjardins } \\
\text { et al. }[27]\end{array}$ & $\begin{array}{c}\text { Omuro } \\
\text { et al. [20] }\end{array}$ \\
\hline Treatment related death & & 9 & & 3 & \\
\hline Hypokalemia & 7.5 & & & & 3 \\
\hline Hypernatremia & 12.5 & & & & \\
\hline Hyponatremia & & & 1 & & 5 \\
\hline Dehydration & & & 1 & 3 & \\
\hline Fatigue & 5 & 9 & 4 & 9 & 5 \\
\hline Thrombocytopenia & & & 13 & & 3 \\
\hline Neutropenia & & & 17 & & \\
\hline Lymphopenia & & & & & 30 \\
\hline Anemia & & & 3 & & \\
\hline Dyspnea & & & & 3 & \\
\hline Diarrhea & & & & 3 & \\
\hline Somnolence & 2.5 & & & & \\
\hline Vomiting & & & 1 & & \\
\hline Colitis & & & & 3 & \\
\hline Pancreatitis & & & & 3 & \\
\hline Anal fissure & & 4 & & & \\
\hline Hemorrhoids & & & & 3 & \\
\hline Transaminase elevation & & & 1 & & 5 \\
\hline Stomatitis & & & 1 & & \\
\hline Thromboembolic event & & 17 & 1 & & 3 \\
\hline Neuropathy & & & & & 3 \\
\hline Allergic reaction & & 4 & & & \\
\hline Infection & & & & & 3 \\
\hline Hypophosphatemia & & & 1 & & \\
\hline
\end{tabular}


PR and 25\% SD. The survival data indicated PFS at 6 months 19\%, median PFS of 2 months and median OS of 7 months. One-year OS was 35\%. The treatment was associated with tolerable toxicity, but 30\% of patients developed lymphopenia.

\section{Conclusion}

The study reached the goal with 4 cases of CR and PR in the ITT population. The data acquired in patients with RGBM present improved survival outcomes due to ANP therapy, and tolerable toxicity. The future studies will contain control groups.

\section{Acknowledgements}

The authors express their appreciation to the additional physicians involved in the care of the patients: Drs. Robert A. Weaver, Robert I. Lewy, Eva Kubove, and Barbara Szymkowski. Preparation of the manuscript was provided by Carolyn Powers, Adam Golunski, and Jennifer Pineda.

\section{Conflict of Interest}

S. R. Burzynski, M.D., Ph.D. is Chairman of the Board of Directors and President of Burzynski Research Institute, Inc. G.S.B. is a member of the Board of Directors of Burzynski Research Institute, Inc. T.J.J. is the Vice President of Clinical Trials at Burzynski Research Institute, Inc. All authors are employed by Burzynski Clinic.

\section{References}

[1] Ostrom, Q.T., Gittleman, H., Farah, P., Ondracek, A., Chen, Y., Wolinsky, Y., et al. (2013) CBTRUS Statistical Report: Primary Brain and Central Nervous System Tumors Diagnosed in the United States in 2006-2010. Neuro-Oncology, 15, ii1-ii56. http://dx.doi.org/10.1093/neuonc/not151

[2] Fisher, J.L., Schwartzbaum, J., Wrensch, M. and Wiemels, J.L. (2007) Epidemiology of Brain Tumors. Neurologic Clinics, 25, 867-890. http://dx.doi.org/10.1016/j.ncl.2007.07.002

[3] Wrensch, M., Lee, M., Miike, R., et al. (1997) Familial and Personal Medical History of Cancer and Nervous System Conditions among Adults with Glioma and Controls. American Journal of Epidemiology, 145, 581-593. http://dx.doi.org/10.1093/oxfordjournals.aje.a009154

[4] Preston, D.L., Ron, E., Yonehara, S., et al. (2002) Tumors of the Nervous System and Pituitary Gland Associated with Atomic Bomb Radiation Exposure. Journal of the National Cancer Institute, 94, 1555-1563. http://dx.doi.org/10.1093/jnci/94.20.1555

[5] (1982) Brain Tumors in the Chemical Industry. Annals of the New York Academy of Sciences, 381, 1-362.

[6] National Toxicology Program (1978) Bioassay of 1H-Benzotriazole for Possible Carcinogenicity. National Cancer Institute Carcinogenesis Technical Report Series, 88, 1-131.

[7] Sills, R.C., Hailey, J.R., Neal, J., Boorman, G.A., Haseman, J.K. and Melnick, R.L. (1999) Examination of Low-Incidence Brain Tumor Responses in F344 Rats Following Chemical Exposures in National Toxicology Program Carcinogenicity Studies. Toxicologic Pathology, 27, 589-599. http://dx.doi.org/10.1177/019262339902700513

[8] U.S. Department of Labor OSHA (2007) Occupational Safety and Health Guideline for Glycidol. Washington, D.C.

[9] Turner, M.C., Chen, Y., Krewski, D. and Ghadirian, P. (2006) An Overview of the Association between Allergy and Cancer. International Journal of Cancer, 118, 3124-3132. http://dx.doi.org/10.1002/ijc.21752

[10] Wrensch, M., Wiencke, J.K., Wiemels, J., et al. (2006) Serum IgE, Tumor Epidermal Growth Factor Receptor Expression, and Inherited Polymorphisms Associated with Glioma Survival. Cancer Research, 66, 4531-4541. http://dx.doi.org/10.1158/0008-5472.CAN-05-4032

[11] Hanahan, D. and Weinberg, R.A. (2000) The Hallmarks of Cancer. Cell, 100, 57-70. http://dx.doi.org/10.1016/S0092-8674(00)81683-9

[12] Sturm, D., Witt, H., Hovestadt, V., Khuong-Quang, D.A., Jones, D.T.W., Konermann, C., et al. (2012) Hotspot Mutations in H3F3A and IDH1 Define Distinct Epigenetic and Biological Subgroups of Glioblastoma. Cancer Cell, 22, 425437. http://dx.doi.org/10.1016/j.ccr.2012.08.024

[13] Burzynski, S.R. and Patil, S.S. (2014) The Effect of Antineoplastons A10 and AS2-1 and Metabolites of Sodium Phenylbutyrate on Gene Expression in Glioblastoma Multiforme. Journal of Cancer Therapy, in press.

[14] Burzynski, S.R., Burzynski, G.S. and Janicki, T.J. (2014) Recurrent Glioblastoma Multiforme, a Strategy for Long- 
Term Survival. Journal of Cancer Therapy, in press.

[15] Stupp, R., Mason, W.P., van den Bent, M.J., Weller, M., Fisher, B., Taphoorn, M.J.B., et al. (2005) Radiotherapy plus Concomitant and Adjuvant Temozolomide for Glioblastoma. New England Journal of Medicine, 352, 987-996. http://dx.doi.org/10.1056/NEJMoa043330

[16] Hegi, M.E., Diserens, A.C., Gorlia, T., Hamou, M.F., de Tribolet, N., Weller, M., et al. (2005) MGMT Gene Silencing and Benefit from Temozolomide in Glioblastoma. New England Journal of Medicine, 352, 997-1003. http://dx.doi.org/10.1056/NEJMoa043331

[17] Gilbert, M.R., Wang, M., Aldape, K.D., Stupp, R., Hegi, M., Jaeckle, K.A., et al. (2011) RTOG 0525: A Randomized Phase III Trial Comparing Standard Adjuvant Temozolomide (TMZ) with a Dosedense (dd) Schedule in Newly Diagnosed Glioblastoma (GBM). Journal of Clinical Oncology, 29, 2006.

[18] National Comprehensive Cancer Network. NCCN Guidelines: Central Nervous System Cancers. http://www.nccn.org/professionals/physician_gls/f_guidelines.asp

[19] Weller, M., Cloughesy, T., Perry, J.R. and Wick, W. (2013) Standards of Care for Treatment of Recurrent Glioblastoma-Are We There Yet? Neuro-Oncology, 15, 4-27. http://dx.doi.org/10.1093/neuonc/nos273

[20] Omuro, A., Chan, T.A., Abrey, L.E., Khasraw, M., Reiner, A.S., Kaley, T.J., et al. (2013) Phase II Trial of Continuous Low-Dose Temozolomide for Patients with Recurrent Malignant Glioma. Neuro-Oncology, 15, 242-250. http://dx.doi.org/10.1093/neuonc/nos295

[21] Burzynski, S.R. (2004) The Present State of Antineoplaston Research (1). Integrative Cancer Therapies, 3, 47-58. http://dx.doi.org/10.1177/1534735403261964

[22] Burzynski, S.R., Conde, A.B., Peters, A., Saling, B., Ellithorpe, R., Dougherty, J.P. and Nacht, C.H. (1999) A Retrospective Study of Antineoplastons A10 and AS2-1 in Primary Brain Tumours. Clinical Drug Investigation, 18, 1-10. http://dx.doi.org/10.2165/00044011-199918010-00001

[23] Wen, P.Y., Macdonald, D.R., Reardon, D.A., Cloughesy, T.F., Sorensen, A.G., Galanis, E., et al. (2010) Updated Response Assessment Criteria for High-Grade Gliomas: Response Assessment in Neuro-Oncology Working Group. Journal of Clinical Oncology, 28, 1963-1972. http://dx.doi.org/10.1200/JCO.2009.26.3541

[24] Chang, S.M., Kuhn, J.G., Robins, H.I., Schold, S.C., Spence, A.M., Berger, M.S., Mehta, M.P., Bozik, M.E., Pollack, I., Schiff, D., Gilbert, M., Rankin, C. and Prados, M.D. (1999) Phase II Study of Phenylacetate in Patients with Recurrent Malignant Glioma: A North American Brain Tumor Consortium Report. Journal of Clinical Oncology, 17, 984990.

[25] Vredenburgh, J.J., Desjardins, A., Herndon, J.E., Dowell, J.M., Reardon, D.A., Quinn, J.A., et al. (2007) Phase II Trial of Bevacizumab and Irinotecan in Recurrent Malignant Glioma. Clinical Cancer Research, 13, 1253-1259. http://dx.doi.org/10.1158/1078-0432.CCR-06-2309

[26] Reardon, D.A., Desjardins, A., Peters, K.B., Vredenburgh, J.J., Gururangan, S., Sampson, J.H., McLendon, R.E., Herndon II, J.E., Coan, A., Threatt, S., Friedman, A.H. and Friedman, H.S. (2011) Phase 2 Study of Carboplatin, Irinotecan, and Bevacizumab for Recurrent Glioblastoma after Progression on Bevacizumab Therapy. Cancer, 117, 53515358. http://dx.doi.org/10.1002/cncr.26188

[27] Desjardins, A., Reardon, D.A., Coan, A., Marcello, J., Herndon II, J.E., Bailey, L., Peters, K.B., Friedman, H.S. and Vredenburgh, J.J. (2012) Bevacizumab and Daily Temozolomide for Recurrent Glioblastoma. Cancer, 118, $1302-1312$. http://dx.doi.org/10.1002/cncr.26381 
Scientific Research Publishing (SCIRP) is one of the largest Open Access journal publishers. It is currently publishing more than 200 open access, online, peer-reviewed journals covering a wide range of academic disciplines. SCIRP serves the worldwide academic communities and contributes to the progress and application of science with its publication.

Other selected journals from SCIRP are listed as below. Submit your manuscript to us via either submit@scirp.org or Online Submission Portal.
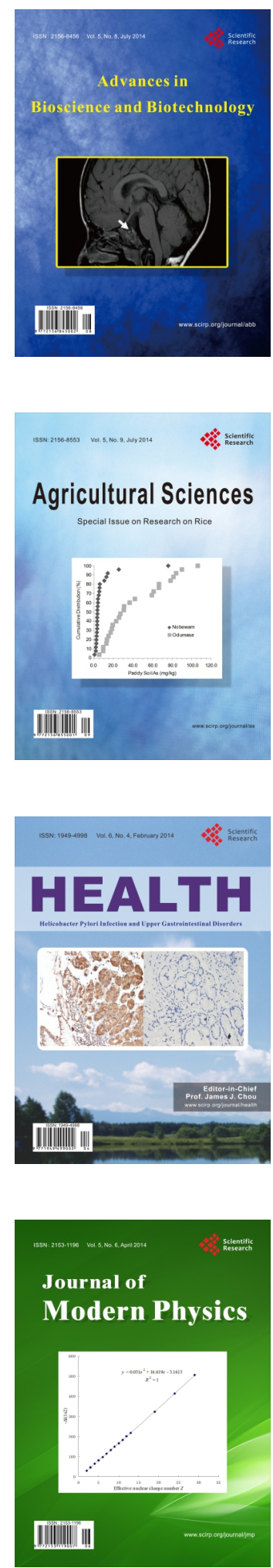
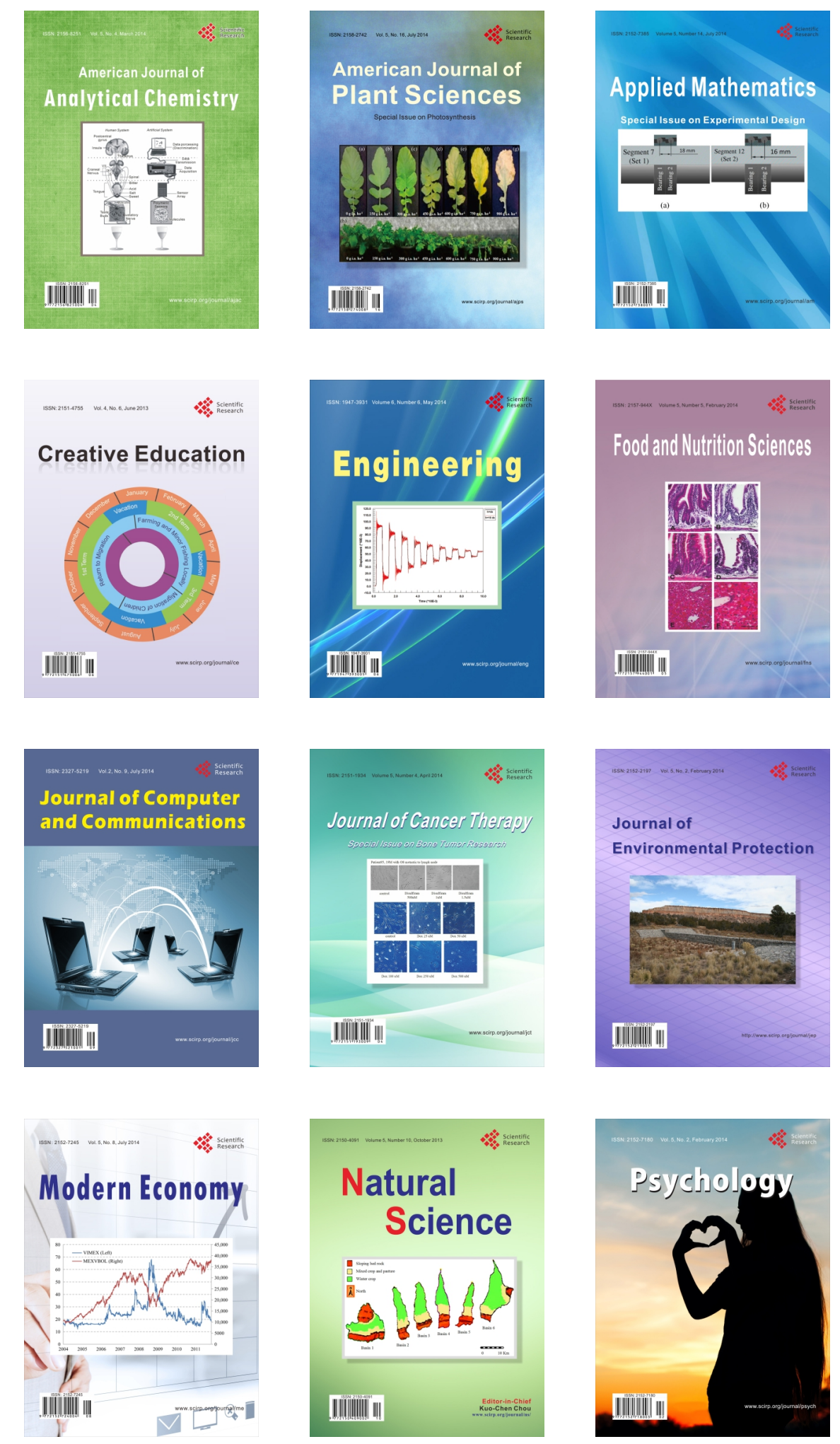